\title{
Fixed points of two-degree of freedom systems
}

\author{
Mohammed Abu-Hilal \\ Department of Mechanical and Industrial \\ Engineering, Applied Science University, \\ Amman 11931, Jordan
}

Received 7 April 1997

Revised 11 May 1998

The presence of fixed points in a frequency response of vibrating systems can greatly complicate the vibration reduction if these points are not recognized.

In this paper, the fixed points of two-degree of freedom systems are studied. The frequencies at which fixed points occur and their amplitudes are determined analytically.

\section{Introduction}

In general, vibrations are undesirable. Their effects on mechanical systems are injurious and can cause costly failures. Therefore, vibrations and their effects need to be suppressed.

A phenomenon which can strongly complicate vibration reduction is the occurrence of fixed points in a frequency response. These points are only recognized if the system parameters are varied. There are three types of fixed points: damping, mass, and stiffness. Fixed points, which occur in a frequency response during a parametric variation of damping, are called damping fixed points. At the frequencies where these points occur, the vibration amplitudes remain constant regardless of the damping values.

Mass and stiffness fixed points are defined in a similar manner. These points occur perfectly in a frequency response only in the absence of damping. Therefore, an undamped system is assumed by their determination.

Damping fixed points were treated by Den Hartog [2], Klotter [5], and Dimarogonas and Haddad [3] in connection with vibration isolation and vibration absorption of systems with a single degree of freedom. In vibration isolation, one fixed point occurs at the frequency $\omega=\sqrt{\omega_{\mathrm{n}}}$, where $\omega_{\mathrm{n}}$ is the natural frequency

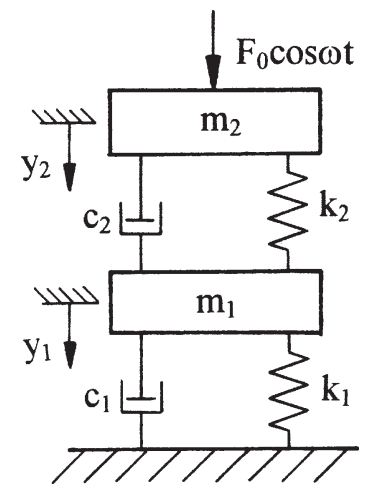

Fig. 1. Two-degree of freedom system.

of the system. In vibration absorption, two fixed points occur in the frequency response of the primary mass and three fixed points occur in the frequency response of the absorber. Also, Bogy and Paslay [1] and Henny and Raney [4] have used the damping fixed points to obtain optimal damping. To our knowledge, however, mass and stiffness fixed points have not been explored previously.

In this paper, a linear system with two degrees of freedom, as shown in Fig. 1, is presented. It is excited by a harmonic force $F(t)=F_{0} \cos \omega t$, that acts on a mass, $m_{2}$. All fixed points, which occur in this system in the frequency responses of the vibrating masses $m_{1}$ and $m_{2}$, and the frequency response of the force transmitted to the base will be discussed in this paper. The frequencies at which these fixed points occur and their amplitudes will be determined analytically. Also, their effect on vibration reduction will be discussed.

\section{Equations of motion}

The equations of motion of the system shown in Fig. 1 are:

$$
\begin{aligned}
& m_{1} \ddot{y}_{1}+c_{1} \dot{y}_{1}+c_{2}\left(\dot{y}_{1}-\dot{y}_{2}\right) \\
& \quad+k_{1} y_{1}+k_{2}\left(y_{1}-y_{2}\right)=0, \\
& m_{2} \ddot{y}_{2}+c_{2}\left(\dot{y}_{2}-\dot{y}_{1}\right)+k_{2}\left(y_{2}-y_{1}\right)=F_{0} \cos \omega t .
\end{aligned}
$$


Substituting the steady-state solution

$$
y_{i}(t)=Y_{i} \cos \left(\omega t-\phi_{i}\right), \quad i=1,2
$$

into Eqs (1) and (2), and solving for the amplitudes $Y_{i}$, yields

$Y_{1}=\frac{F_{0}}{\Delta} \sqrt{k_{2}^{2}+\left(c_{2} \omega\right)^{2}}$,

$Y_{2}=\frac{F_{0}}{\Delta} \sqrt{\left(k_{1}+k_{2}-m_{1} \omega^{2}\right)^{2}+\left(c_{1}+c_{2}\right)^{2} \omega^{2}}$,

where

$$
\begin{aligned}
\Delta= & {\left[\left\{m_{1} m_{2} \omega^{4}-\left[k_{1} m_{2}+k_{2}\left(m_{1}+m_{2}\right)+c_{1} c_{2}\right] \omega^{2}\right.\right.} \\
& \left.+k_{1} k_{2}\right\}^{2}+\left\{\left(k_{1} c_{2}+k_{2} c_{1}\right) \omega\right. \\
& \left.\left.-\left[m_{1} c_{2}+m_{2}\left(c_{1}+c_{2}\right)\right] \omega^{3}\right\}^{2}\right]^{1 / 2}
\end{aligned}
$$

$Y_{1}$ and $Y_{2}$ are the vibration amplitudes of the masses $m_{1}$ and $m_{2}$, respectively. The force transmitted $F_{\text {Tr }}$ to the base is determined by

$$
F_{\mathrm{Tr}}=c_{1} \dot{y}+k_{1} y_{1}=F_{\mathrm{T}} \cos (\omega t-\psi),
$$

where the amplitude of the force transmitted is given by

$$
F_{\mathrm{T}}=\frac{F_{0}}{\Delta}\left(\left(k_{1} k_{2}-c_{1} c_{2} \omega^{2}\right)^{2}+\left[\left(k_{1} c_{2}+k_{2} c_{1}\right) \omega\right]^{2}\right)^{1 / 2}
$$

\section{Mass fixed points}

By varying the values of $m_{1}$ while all other parameters of the undapmed system remain constant, all curves of the amplitudes $Y_{1}$ and $F_{\mathrm{T}}$ pass through a mass fixed point, independent of the values of $m_{1}$. This fixed point is determined by equating $Y_{1}$ or $F_{\mathrm{T}}$ to two different values of $m_{1}$. Equating $Y_{1}$ for the values $m_{1}=0$ and $m_{1}=1$ yields that this fixed point occurs at the frequency

$$
\omega_{m, 1}=\sqrt{k_{2} / m_{2}}
$$

where the values of $Y_{1}$ and $F_{\mathrm{T}}$ at the aforementioned frequency are obtained from Eqs (4) and (8), respectively, as:

$$
\begin{aligned}
& Y_{1}\left(\omega_{m, 1}\right)=F_{0} / k_{2}, \\
& F_{\mathrm{T}}\left(\omega_{m, 1}\right)=\left(k_{1} / k_{2}\right) F_{0} .
\end{aligned}
$$

Since these values are independent of $m_{1}$, then by their determination values of $m_{1}$, which simplify Eqs (4) and (8) are selected. Usually, the values 0 and/or $\infty$ are selected. The control of the amplitudes $Y_{1}$ and $F_{\mathrm{T}}$ at the working frequencies close to $\omega_{m, 1}$ can not be succeeded by varying the value of $m_{1}$.

On the other hand, by varying the values of $m_{2}$, all curves of the amplitudes $Y_{1}$ and $F_{\mathrm{T}}$ pass through a mass fixed point, independent of the values of $m_{2}$. This fixed point can be determined as previously described and is located at the frequency

$$
\omega_{m, 2}=\sqrt{\left(k_{1}+k_{2}\right) / m_{1}},
$$

where the amplitudes $Y_{1}$ and $F_{\mathrm{T}}$ at this frequency are determined from Eqs (4) and (8), respectively, as:

$$
\begin{aligned}
& Y_{1}\left(\omega_{m, 2}\right)=F_{0} / k_{2}, \\
& F_{\mathrm{T}}\left(\omega_{m, 2}\right)=\left(k_{1} / k_{2}\right) F_{0} .
\end{aligned}
$$

These amplitudes, which are independent of $m_{2}$, cannot be controlled by varying the values of $m_{2}$.

The frequency response of the mass $m_{2}$ does not possess mass fixed points neither by varying the values of $m_{1}$ nor the values of $m_{2}$. However, all curves of the amplitude $Y_{2}$ become zero at the absorber frequency $\omega_{\mathrm{a}}=\sqrt{\left(k_{1}+k_{2}\right) / m_{1}}$, regardless of the values of $m_{2}$.

\section{Stiffness fixed points}

By varying the values of $k_{1}$ of the undamped system, the following outcomes are achieved:

1. The curves of $Y_{1}$ possess a stiffness fixed point at the frequency

$$
\omega_{k, 1}=\sqrt{k_{2} / m_{2}}
$$

where the amplitude $Y_{1}$ at this frequency is obtained from Eq. (4) as:

$$
Y_{1}\left(\omega_{k, 1}\right)=F_{0} / k_{2} .
$$


2. The curves of $F_{\mathrm{T}}$ possess a stiffness fixed point at the frequency

$$
\omega_{k, 2}=\sqrt{\frac{k_{2}\left(m_{1}+m_{2}\right)}{m_{1} m_{2}}},
$$

where the amplitude $F_{\mathrm{T}}$ at this frequency is obtained from Eq. (8) as:

$$
F_{\mathrm{T}}\left(\omega_{k, 2}\right)=\left(m_{1} / m_{2}\right) F_{0} .
$$

3. The reduction of $Y_{1}\left(\omega_{k, 1}\right)$ and $F_{\mathrm{T}}\left(\omega_{k, 2}\right)$ will not succeed by varying the values of $k_{1}$, since these amplitudes are independent of the values of $k_{1}$.

4. The curves of the amplitude $Y_{2}$ possess no fixed points.

On the other hand, by varying the values of $k_{2}$ of the undamped system, the curves of all three amplitudes, namely, $Y_{1}, Y_{2}$, and $F_{\mathrm{T}}$ possess the same stiffness fixed point. This fixed point occurs at the frequency

$$
\omega_{k, 3}=\sqrt{k_{1} / m_{1}},
$$

where the amplitudes $Y_{1}, Y_{2}$, and $F_{\mathrm{T}}$ at this frequency are obtained from Eqs (4), (5), and (8), respectively, as:

$$
\begin{aligned}
Y_{1}\left(\omega_{k, 3}\right) & =\frac{m_{1}}{k_{1} m_{2}} F_{0}, \\
Y_{2}\left(\omega_{k, 3}\right) & =\frac{m_{1}}{k_{1} m_{2}} F_{0}, \\
F_{\mathrm{T}}\left(\omega_{k, 3}\right) & =\frac{m_{1}}{m_{2}} F_{0} .
\end{aligned}
$$

These amplitudes cannot be reduced by varying the values of $k_{2}$.

\section{Damping fixed points}

By varying the values of $c_{1}$ in the absence of $c_{2}$, the following outcomes are achieved:

1. The curves of $Y_{1}$ possess only one damping fixed point, which occurs at the frequency

$$
\omega_{c, 1}=\sqrt{k_{2} / m_{2}},
$$

where $Y_{1}$ at this frequency is obtained from Eq. (4) as:

$$
Y_{1}\left(\omega_{c, 1}\right)=F_{0} / k_{2} .
$$

2. The curves of $Y_{2}$ possess two damping fixed points which are located at the frequencies

$$
\begin{aligned}
& \omega_{c, 2,3}=\frac{1}{\sqrt{2 m_{1} m_{2}}}\left[k_{1} m_{2}+k_{2}\left(m_{1}+m_{2}\right)\right. \\
& \left.\mp\left(\left(k_{1} m_{2}-k_{2} m_{1}\right)^{2}+m_{2}^{2} k_{2}\left(2 k_{1}+k_{2}\right)\right)^{1 / 2}\right]^{1 / 2},
\end{aligned}
$$

where $Y_{2}$ at these frequencies is obtained from Eq. (5) as:

$$
Y_{2}\left(\omega_{c, i}\right)=\frac{F_{0}}{k_{2}-m_{2} \omega_{c, i}^{2}}, \quad i=2,3 .
$$

3. The curves of $F_{\mathrm{T}}$ possess three damping fixed points. These points are located at the frequencies

$$
\begin{aligned}
\omega_{c, 4,5}= & \frac{1}{\sqrt{2 m_{1} m_{2}}}\left[2 k_{1} m_{2}+k_{2}\left(m_{1}+m_{2}\right)\right. \\
& \mp\left(\left[2 k_{1} m_{2}+k_{2}\left(m_{1}+m_{2}\right)\right]^{2}\right. \\
& \left.\left.-8 k_{1} k_{2} m_{1} m_{2}\right)^{1 / 2}\right]^{1 / 2}, \\
\omega_{c, 6}= & \sqrt{\frac{k_{2}\left(m_{1}+m_{2}\right)}{m_{1} m_{2}}},
\end{aligned}
$$

where $F_{\mathrm{T}}$ at these frequencies is obtained from Eq. (8) as:

$$
F_{\mathrm{T}}\left(\omega_{c, i}\right)=\frac{F_{0} k_{2}}{k_{2}-m_{2} \omega_{c, i}^{2}}, \quad i=4,5,6
$$

By varying the values of $c_{2}$ in the absence of $c_{1}$ yields that the curves of $Y_{1}, Y_{2}$, and $F_{\mathrm{T}}$ possess three damping fixed points. The fixed points of $Y_{1}$ and $F_{\mathrm{T}}$ are located at the frequencies

$$
\begin{aligned}
\omega_{c, 7,8}= & \frac{1}{\sqrt{2 m_{1} m_{2}}}\left[k_{1} m_{2}+2 k_{2}\left(m_{1}+m_{2}\right)\right. \\
& \mp\left(\left[k_{1} m_{2}+2 k_{2}\left(m_{1}+m_{2}\right)\right]^{2}\right. \\
& \left.\left.-8 k_{1} k_{2} m_{1} m_{2}\right)^{1 / 2}\right]^{1 / 2} \\
\omega_{c, 9}= & \sqrt{k_{1} / m_{1}}
\end{aligned}
$$

where $Y_{1}$ and $F_{\mathrm{T}}$ at these frequencies are determined by using Eqs (4) and (8), respectively, as: 


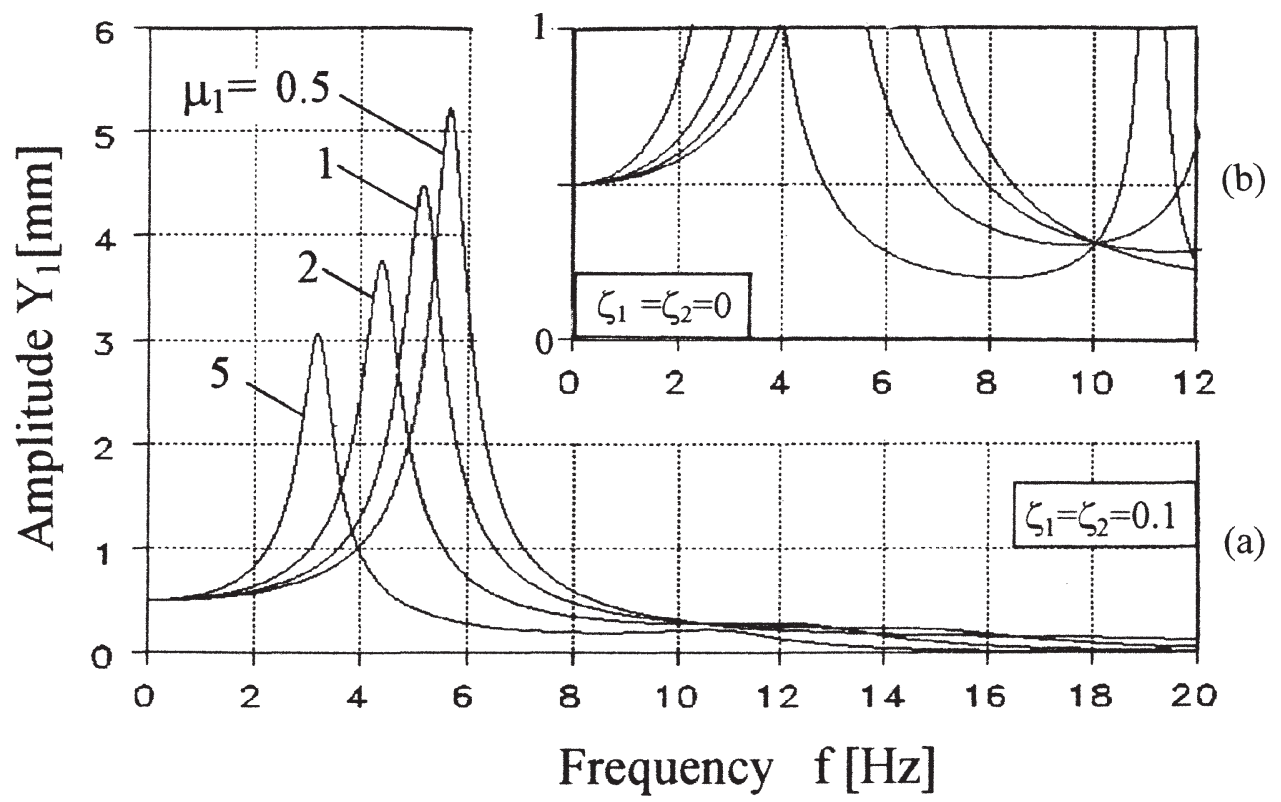

Fig. 2. Frequency response for different values of $\mu_{1}=m_{1} /(80 \mathrm{~kg}$ ): (a) damped, (b) undamped.

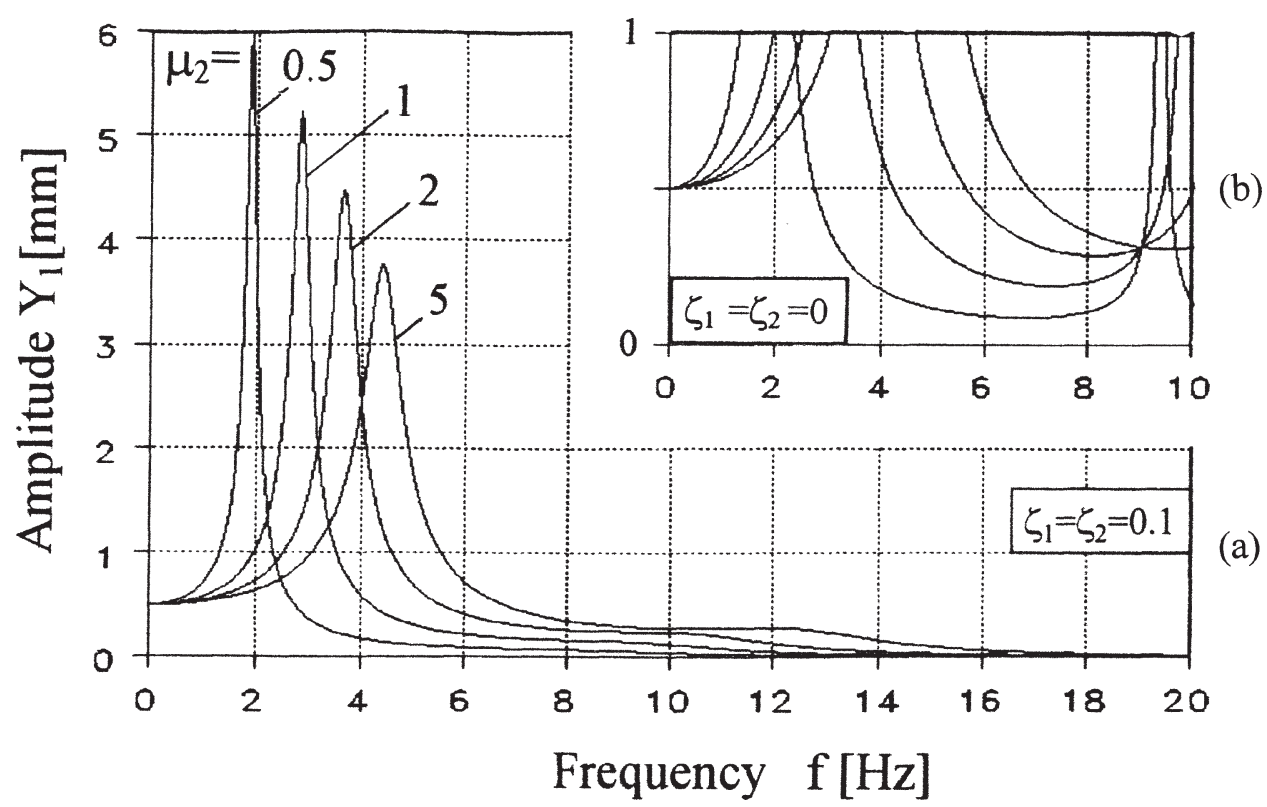

Fig. 3. Frequency response for different values of $\mu_{2}=m_{2} /(160 \mathrm{~kg}$ ): (a) damped, (b) undamped.

$$
\begin{aligned}
& Y_{1}\left(\omega_{c, i}\right)=\frac{F_{0}}{k_{1}-\left(m_{1}+m_{2}\right) \omega_{c, i}^{2}}, i=7,8,9, \\
& F_{\mathrm{T}}\left(\omega_{c, i}\right)=\frac{k_{1} F_{0}}{k_{1}-\left(m_{1}+m_{2}\right) \omega_{c, i}^{2}}, i=7,8,9 .
\end{aligned}
$$

The fixed points of $Y_{2}$ are located at the frequencies

$$
\begin{aligned}
& \omega_{c, 10,11}=\frac{1}{\sqrt{m_{1}^{2}+2 m_{1} m_{2}}}\left[\left(k_{1}+k_{2}\right)\left(m_{1}+m_{2}\right)\right. \\
& \mp\left(\left[\left(k_{1}+k_{2}\right)\left(m_{1}+m_{2}\right)\right]^{2}\right. \\
& \left.\left.-\left(2 k_{1} k_{2}+k_{1}^{2}\right)\left(m_{1}^{2}+2 m_{1} m_{2}\right)\right)^{1 / 2}\right]^{1 / 2} \\
& \omega_{c, 12}=\sqrt{k_{1} / m_{1}}
\end{aligned}
$$




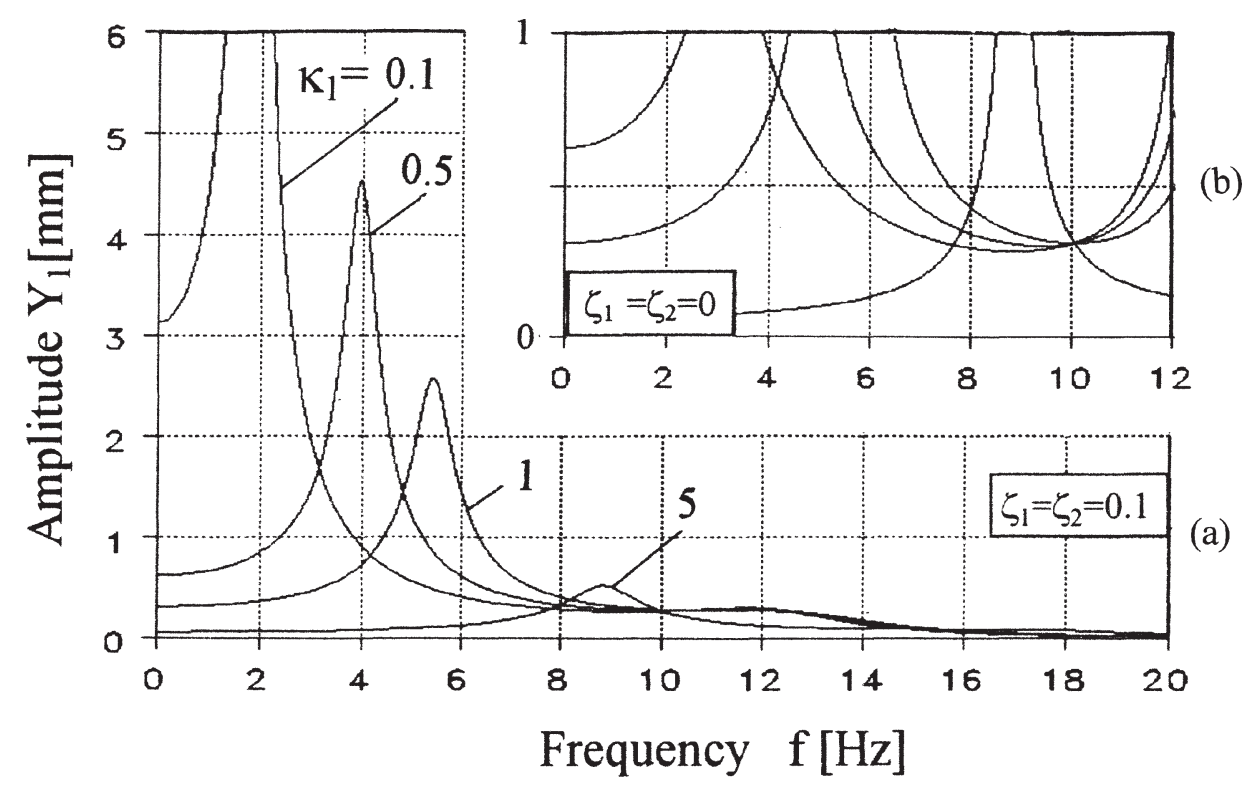

Fig. 4. Frequency response for different values of $\kappa_{1}=k_{1} /(320 \mathrm{kN} / \mathrm{m})$ : (a) damped, (b) undamped.

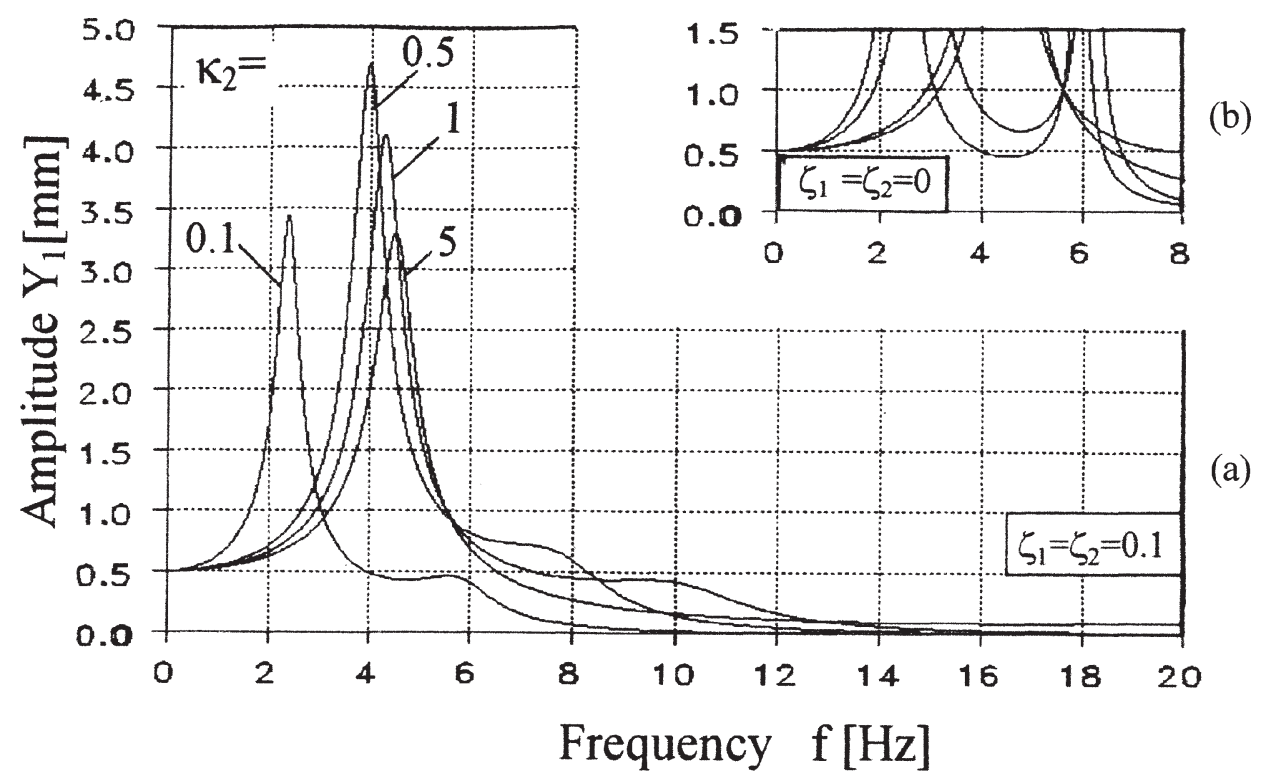

Fig. 5. Frequency response for different values of $\kappa_{2}=k_{2} /(200 \mathrm{kN} / \mathrm{m})$ : (a) damped, (b) undamped.

where $Y_{2}$ at these frequencies is obtained from Eq. (5) as:

$$
Y_{2}\left(\omega_{c, i}\right)=\frac{F_{0}}{k_{1}-\left(m_{1}+m_{2}\right) \omega_{c, i}^{2}}, i=10,11,12
$$

When the working frequencies are near damping fixed points, the vibration amplitudes can only be slightly influenced by varying the values of $c_{i}(i=1,2)$. In this case, isolators and/or absorbers can be used for vibration reduction.

\section{Numerical example}

To verify the above model, a numerical example will be presented. It is based on the following data: $m_{1}=$ 


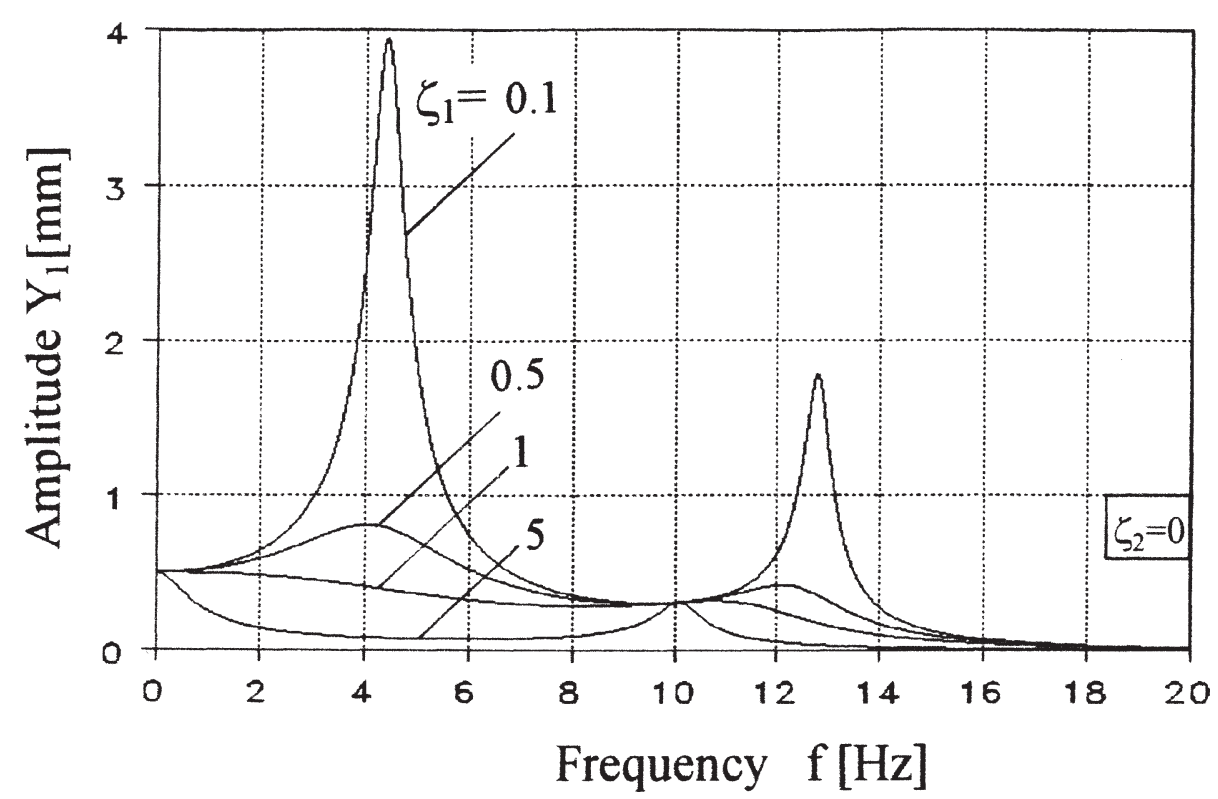

Fig. 6. Frequency response for different values of $\zeta_{1}=c_{1} /(11314 \mathrm{~N} \mathrm{~s} / \mathrm{m})$.

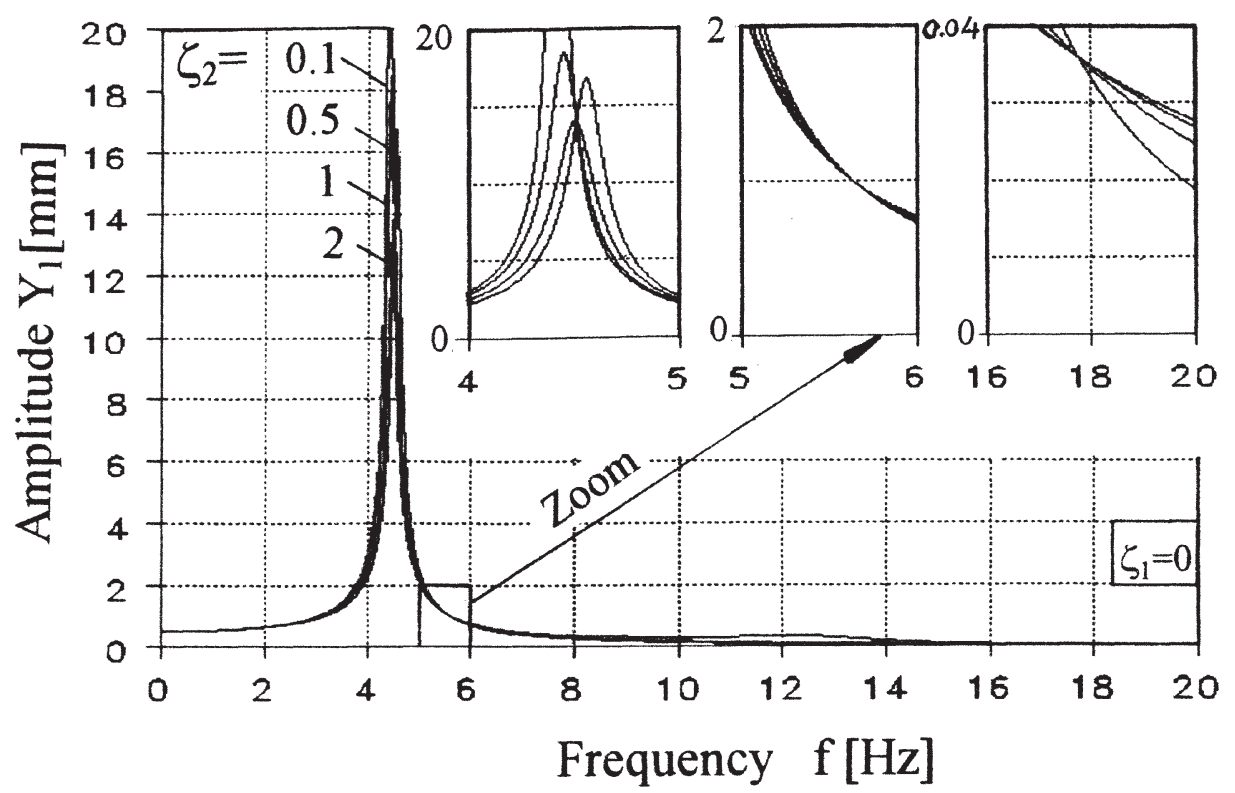

Fig. 7. Frequency response for different values of $\zeta_{2}=c_{2} /(10119 \mathrm{~N} \mathrm{~s} / \mathrm{m})$.

$160 \mathrm{~kg}, m_{2}=80 \mathrm{~kg}, k_{1}=200 \mathrm{kN} / \mathrm{m}, k_{2}=320 \mathrm{kN} / \mathrm{m}$, $c_{1}=1131 \mathrm{~N} \mathrm{~s} / \mathrm{m}, c_{2}=1012 \mathrm{~N} \mathrm{~s} / \mathrm{m}, F_{0}=100 \mathrm{~N}$.

For convenience, the following nondimensional parameters are used: $\mu_{1}=m_{1} / m_{2}, \mu_{2}=m_{2} / m_{1}$, $\kappa_{1}=k_{1} / k_{2}, \kappa_{2}=k_{2} / k_{1}, \zeta_{1}=c_{1} / 2 \sqrt{k_{1} m_{1}}, \zeta_{2}=$ $c_{2} / 2 \sqrt{k_{2} m_{2}}$.

Representatively, only the frequency responses of $m_{1}$ will be discussed. On the other hand, the frequency responses of $m_{2}$ and the force transmitted will not be presented due to the similarity in the discussion.

Figures 2-5 present the frequency responses for the damped (i.e., $\zeta_{1}=\zeta_{2}=0.1$ ), and the undamped (i.e., $\left.\zeta_{1}=\zeta_{2}=0\right)$ systems. Figures 2 and 3 present frequency responses for different values of $\mu_{1}$ and $\mu_{2}$, respectively. While Figs 4 and 5 present frequency responses for different values of $\kappa_{1}$ and $\kappa_{2}$, respectively. 
The frequency responses of the undamped systems enable us to determine the fixed points precisely. However, these points can only be estimated from the frequency responses of the damped systems.

Figures 6 and 7 show the frequency responses for different values of $\zeta_{1}$ and $\zeta_{2}$, respectively. In order to make the fixed points of Fig. 7 clear, the regions of these points are plotted in zoom-windows.

From the previous analysis it is obtained for $Y_{1}$ :

a) Mass fixed points:

$$
\begin{aligned}
& f_{m, 1}=\frac{\omega_{m, 1}}{2 \pi}=10.07 \mathrm{~Hz}, \\
& Y_{1}\left(f_{m, 1}\right)=0.313 \mathrm{~mm}, \\
& f_{m, 2}=9.07 \mathrm{~Hz}, \quad Y_{1}\left(f_{m, 2}\right)=0.313 \mathrm{~mm} .
\end{aligned}
$$

b) Stiffness fixed points:

$$
\begin{array}{ll}
f_{k, 1}=10.07 \mathrm{~Hz}, & Y_{1}\left(f_{k, 1}\right)=0.313 \mathrm{~mm}, \\
f_{k, 3}=5.63 \mathrm{~Hz}, & Y_{1}\left(f_{k, 3}\right)=1 \mathrm{~mm} .
\end{array}
$$

c) Damping fixed points:

$$
\begin{array}{ll}
f_{c, 1}=10.07 \mathrm{~Hz}, & Y_{1}\left(f_{c, 1}\right)=0.313 \mathrm{~mm}, \\
f_{c, 7}=4.51 \mathrm{~Hz}, & Y_{1}\left(f_{c, 7}\right)=13.95 \mathrm{~mm}, \\
f_{c, 8}=17.76 \mathrm{~Hz}, & Y_{1}\left(f_{c, 8}\right)=0.036 \mathrm{~mm}, \\
f_{c, 9}=5.63 \mathrm{~Hz}, & Y_{1}\left(f_{c, 9}\right)=1 \mathrm{~mm} .
\end{array}
$$

Comparison of these values with the plots (i.e., Figs 2-7) yields that they coincide with each other.

\section{Conclusions}

In this paper, different types of fixed points, which can occur for a two-degree of freedom system, are pre- sented. The frequencies at which fixed points occur and their amplitudes are determined analytically. The following can be concluded:

- The presence of fixed points may complicate the reduction of vibrations.

- The presence of fixed points cannot be recognized unless a parametric study is performed.

- When the operating frequency is near a mass fixed point, the amplitudes of vibration cannot be effectively reduced by varying the values of masses.

- When the operating frequency is near a stiffness fixed point, the amplitudes of vibration cannot be effectively reduced by varying the stiffness values.

- When the operating frequency is near a damping fixed point, the amplitudes of vibrations cannot be effectively reduced by varying the damping values.

\section{References}

[1] D.B. Bogy and P.R. Paslay, An evaluation of the fixed point method of vibration analysis for a particular system with initial damping, J. Engineering for Industry, Trans. ASME 85B(3) (1963), 233-236.

[2] J.P. Den Hartog, Mechanical Vibrations, McGraw-Hill, New York, 1956.

[3] A.D. Dimarogonas and S. Haddad, Vibration for Engineers, Prentice Hall, New Jersey, 1992.

[4] A. Henney and J.P. Raney, The optimization of damping of four configuration of vibrating uniform beam, J. Engineering for Industry, Trans. ASME 85B(3) (1963), 259-264.

[5] K. Klotter, Technische Schwingungslehre, Zweiter Band, Schwinger von mehreren Freiheitsgraden, 2nd edn, SpringerVerlag, Berlin/New York, 1981. 

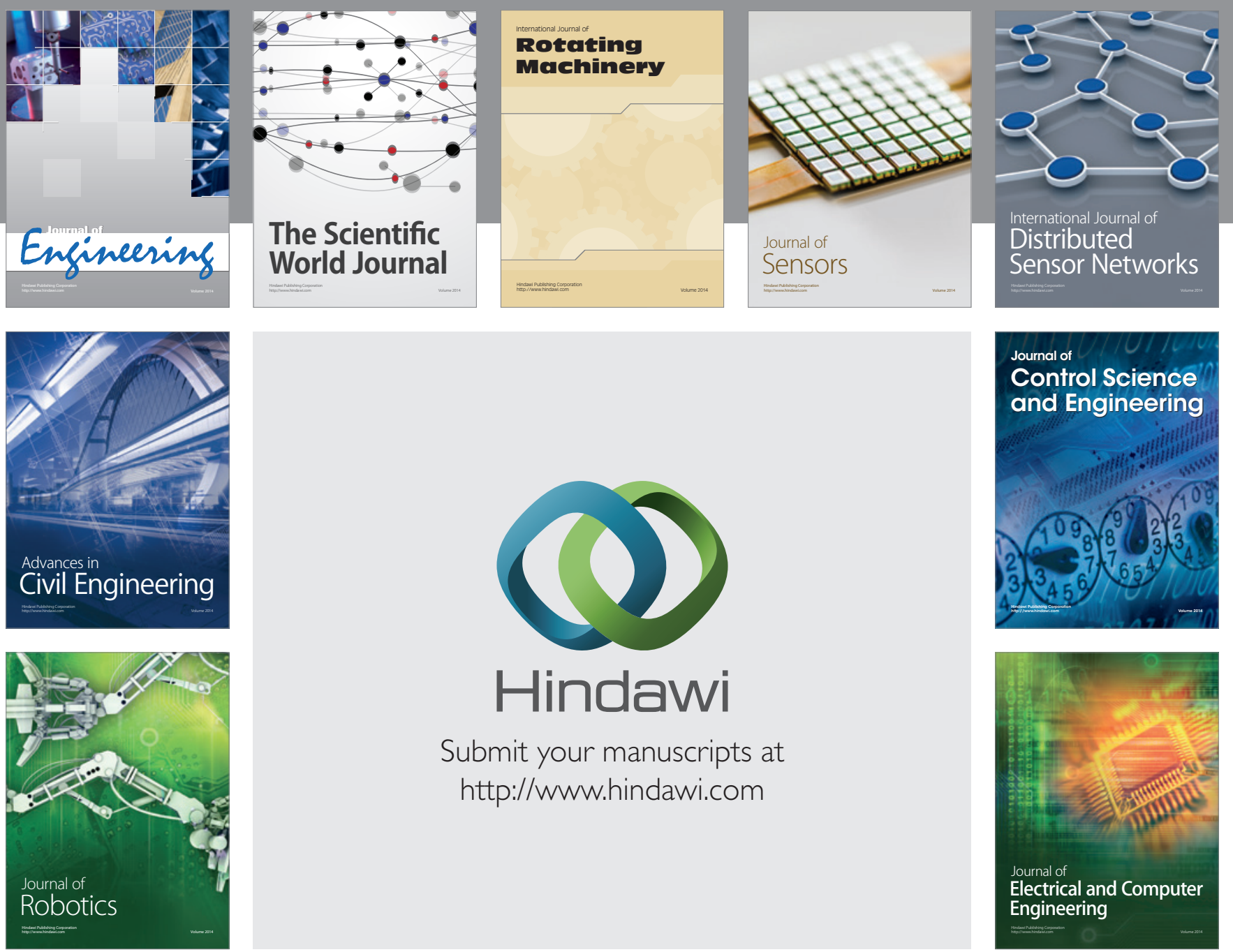

Submit your manuscripts at

http://www.hindawi.com
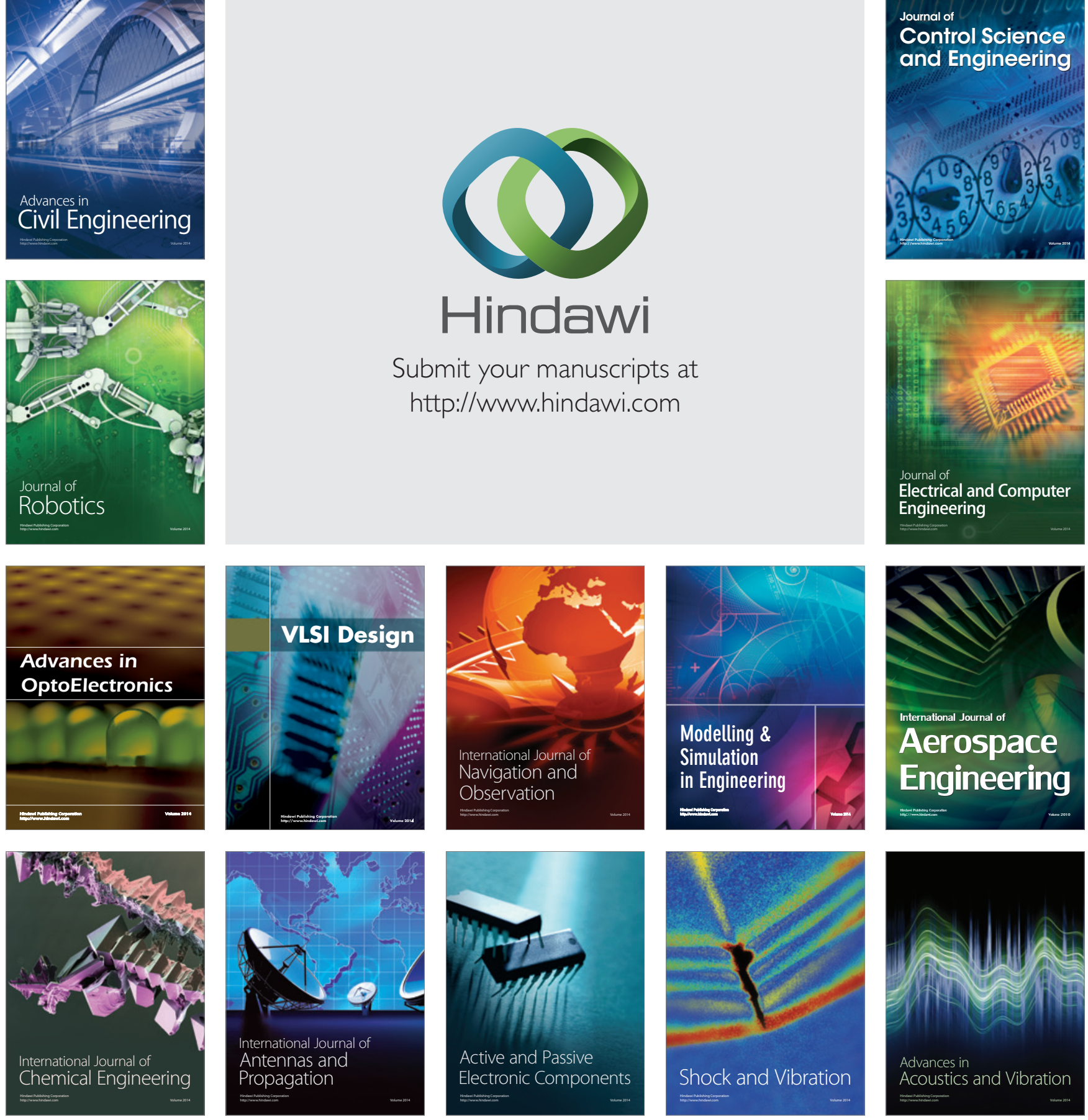